\title{
Cartoneros, recolección informal, ambiente y políticas públicas en Buenos Aires 2001-2012
}

\author{
Scavengers, informal waste collection, public \\ politics in Buenos Aires 2001-2012
}

Verónica Paiva

Centro de Investigaciones Hábitat y Municipio (Ciham), Facultad de Arquitectura, Universidad de Buenos Aires,

licenciatura en Sociología, Universidad Nacional de Mar del Plata, Buenos Aires - Argentina, e-mail: paivav@yahoo.com.ar

\section{Resumen}

El objetivo del artículo es analizar las características del circuito informal de recolección y recuperación residuos que se expandió desde fines de la década del '90 y especialmente hacia el final del año 2001 en la ciudad de Buenos Aires, analizando las razones que provocaron la expansión de dicho circuito, las nuevas características que asume el cirujeo en dicha etapa y las posturas tomadas por la teoría ambiental y las políticas públicas frente a esta forma particular de recuperación de residuos que es prototípica de las ciudades latinoamericanas En forma paralela a la gestión oficial de los residuos se ha extendido una vía informal de recolección y recuperación que es llevado adelante por los sectores pobres de la sociedad que encuentran en la recolección y venta de residuos un camino para la supervivencia cotidiana. El artículo describe las características de dicho circuito informal, las rutas, calles, horarios y tipos de residuos recogidos y analiza el fenómeno no sólo como una expresión de la pobreza urbana sino como un modo de tratamiento de los desechos.

PalaBras-clave: Ambiente. Pobreza urbana. Recolección informal. Políticas públicas.

\section{Abstract}

This paper seeks to analyze the characteristics of the informal circuit of waste collection and recovery in Buenos Aires - developed since the late 1990's and, especially, towards the end of 2001 - and investigate the reasons that stimulate the circuit growth, the new characteristic acquired by the circuit in the second stage, and the positioning taken by environmental theory and public policies about this typology of waste recovery, prototypical in Latin American cities. Parallel to waste management performed by the government, an informal way of waste collection and recovery has been developed, carried out by low income people, who negotiate recyclable waste as a way to survive. The paper outlines the characteristics of this circuit in Buenos Aires - the routes, streets, timetables and types of 
waste - analisyng this phenomenom not only as expression of urban poverty but also as an alternative way to municipal solid waste treatment.

Keywords: Environment. Urban poverty. Informal waste collection. Public policies.

\section{Introducción}

La excesiva generación de residuos producida por el proceso de urbanización, constituye uno de los problemas ambientales más graves de esta época. Para suavizar su impacto, se han ideado diversas estrategias que apuntan a la minimización y valorización de los desechos. La minimización, es decir la reducción al máximo posible de los residuos generados, se alcanza a través de la producción limpia y el consumo sustentable. Por producción limpia deben entenderse un conjunto de disposiciones que implican el uso de tecnologías no contaminantes, la disminución del gasto energético y el empleo de materiales reciclables, mientras que por consumo sustentable se entiende el cambio de hábitos de los ciudadanos hacia estilos de vida menos ávidos de productos ricos en energía y materia.

Por su parte, la valorización, apunta a maximizar el aprovechamiento de los residuos que no puedan dejar de generarse, a través del reuso y el reciclado del material tantas veces como sea posible. Por este camino se intenta hacer realidad el sistema de las tres $\mathrm{R}$ - reducir, recuperar, reciclar - es decir, minimizar la cantidad de desechos que se generan, recuperar el mayor número posible de material y reciclarlo tantas veces como sea factible. La diferencia entre recuperar y reciclar consiste en que mientras en el primer caso sólo se captan los residuos desde la basura sin realizarle ningún tipo de modificación, reciclar implica modificarlos a través de algún sistema físico o químico (RODRÍGUEZ VAL-LLOVERA, 2003, p. 2).

Todas estas estrategias de cuidado ambiental son reiteradamente enunciadas en las diversas conferencias ambientales que se realizan desde la década de 1970 y especialmente desde la Conferencia de las Naciones Unidades sobre el Medio Ambiente y Desarrollo de 1992 (NACIONES UNIDAS - NU, 1992) que fue la que se refirió específicamente al problema del consumo y la producción sustentable y a la gestión integral de los desechos. Sin embargo, llama la atención que todas estas estrategias que se discuten internacionalmente también se pretenden implementar - sin que medie debate alguno - en los países del Tercer Mundo en los cuales, la tan pregonada recuperación de residuos también se produce, aunque por caminos muy diferentes a los recién comentados. En sus ciudades, con baja o nula recolección de residuos, ausencia de sitios adecuados para la disposición final y altos índices de desempleo, son los pobres urbanos los que, por necesidad, se dedican a la recolección y venta de materiales reciclables. Los "hurgadores" en Uruguay, los "pepenadores" en México, los "catadores" en Brasil o los "cartoneros" en Buenos Aires, se han convertido en cuidadores ambientales sin proponérselo, a pesar de la gran pobreza en la que transitan su vida. Se trata de un circuito informal de recuperación en el que intervienen actores con distinto rol y posicionamiento jerárquico - recolectores, intermediarios y empresas finales compradoras de material de postdesecho ${ }^{1}$ - que posee un rasgo particular: ninguno de los actores persigue fines ambientalistas, sino de supervivencia o comercialización. Sin embargo, a partir de su acción se recupera una cantidad más que importante de residuos reutilizables que por esa vía no impactan sobre el ambiente y reingresan al circuito de la producción. Sólo para dar una idea de la importancia de este circuito, vale comentar que en el año 2006 reingresaban por esa vía el 97\% de los desechos recuperados en la ciudad de Buenos Aires (PARDO et al., 2006).

Ante dicho panorama vale efectuarse la siguiente pregunta: ¿qué lectura ha realizado la teoría ambiental frente a un fenómeno como el descripto que se realiza con fines de supervivencia pero genera acciones no intencionales (GIDDENS, 1982) que protegen el ambiente? Y en la misma línea, ¿qué

\footnotetext{
1 Se denomina material de postdesecho a los residuos reutilizables que se generan luego del consumo domiciliario o industrial.
} 
posturas han tomado los organismos encargados de planificar la política de gestión de residuos frente a un fenómeno como el señalado que exhibe la paradoja de proteger el ambiente al tiempo que descuida a quienes contribuyen a preservarlo?

Para responder a estas preguntas, en primer lugar daré cuenta de las causas que impulsaron la expansión del "cirujeo"2 en la ciudad de Buenos Aires hacia fines de 1990, en segundo lugar detallaré algunas de sus características, en tercera instancia me referiré a las políticas de gestión de residuos que se han tomado a nivel del Área Metropolitana de Buenos Aires ${ }^{3}$ y especialmente en la ciudad de Buenos Aires y, por último, intentaré esbozar algunas reflexiones en torno de la cuestión de la teoría ambiental y los canales informales de recolección.

\section{Metodología}

Las reflexiones que se acompañan en este artículo son fruto de una investigación que llevé adelante entre el año 2002 y el 2012. Los métodos usados para recolectar y analizar la información fueron centralmente de tipo cualitativo. Para explorar las formas en que se desplazaban los cartoneros por la ciudad y los modos en que recolectaban los residuos utilicé la observación y la entrevista en profundidad. Tanto en la fase de recolección como en la etapa de análisis de los datos usé el criterio de flexibilidad prototípico de los diseños cualitativos, es decir, fundando la teoría en los datos y trabajando en función de interrogantes abiertos a temas no previstos pero que emergieran durante el trabajo de campo (VASILACHIS DE GIALDINO, 2007).

Para analizar las políticas públicas y las leyes relativas al ordenamiento de la labor cartonera utilicé el análisis de contenido, que muy sucintamente consiste en identificar de manera sistemática, ciertas características específicas dentro de un texto (HOSTIL, 1969).

Realizadas las aclaraciones metodológicas correspondientes a continuación despliego el contenido del artículo.

\section{Causas que motorizaron la expansión del "cirujeo" hacia mitad de la década de 1990}

Si bien el "cirujeo" existió desde antaño en la ciudad de Buenos Aires y el Conurbano, hacia mediados de la década de 1990 se incrementó significativamente la cantidad de personas que ingresaron a la tarea, debido a tres razones. Por un lado, el aumento de la desocupación y la pobreza que afectó a buena parte de la población del Área Metropolitana de Buenos Aires (AMBA), por otro, las falencias de las normativas que regulaban la gestión de los residuos sólidos urbanos en todo el AMBA, y por último, la sustitución de importaciones que comenzó a regir en el país luego de la modificación de la paridad cambiaria a principios del año 2002. Pero además de estos motivos, existen otros factores, como la fuerte crisis social y política que estalló en la Argentina en el año $2001{ }^{\prime \prime}$ y que influyó no solamente en el aumento de la cantidad de personas que ingresaron al "cirujeo", si no en la emergencia de un nuevo sujeto social llamado "cartonero", al que desde la crisis se le asocian funciones ligadas al "reciclaje y la recuperación" de desechos, y a partir de cuya emergencia se sancionan políticas públicas, que intentan incorporarlo a la gestión oficial.

En cuanto a la desocupación, fue el efecto más visible de las diversas políticas económicas que se

\footnotetext{
2 En la ciudad de Buenos Aires los recolectores informales de residuos fueron llamados "cirujas" (en alusión a la palabra cirujano) desde mediados del siglo XIX en que surge el primer barrio formado por personas dedicadas a la recolección informal en el sur de la ciudad y al calor de la incipiente industria que comenzaba a surgir en la ciudad. Dicho barrio se llamó "de las ranas o de las latas" y existió hasta principios del siglo XX (PAIVA, 2008).

3 Ciudad de Buenos Aires y 24 partidos colidantes (INDEC, 2003).

4 Se conoce como "crisis 2001" al conjunto de hechos de orden económico, social y político que eclosionaron a fines de ese año. Por un lado, el congelamiento forzado de los depósitos bancarios que originó protestas masivas en los sectores de clase media, por otro las protestas piqueteras surgidas a partir de la desocupación que afectó a vastos sectores de la población y, por último, la crisis de representación política que incluyó a toda la dirigencia, pero encontró su punto culminante con la renuncia del presidente De la Rúa el 20 de diciembre de 2001.
} 
implementaron durante los años '90 y que tuvieron como corolario el incremento paulatino del desempleo, que creció del 6,35\% en 1990 a un $13,8 \%$ en 1999 y a un $15 \%$ en diciembre de 2001 (BECCARIA, 2001). En forma paralela a estos niveles de desempleo, la pobreza fue creciendo en forma proporcional, ya que se estima que para el año 2000 el 29,7\% de la población estaba bajo la línea de pobreza y en mayo de 2001 el 32,7\% de la población era pobre (INSTITUTO NACIONAL DE ESTADÍSTICA Y CENSO - INDEC, 2007).

Dentro de este cuadro de situación, las limitaciones impuestas por las normativas que regían la gestión pública de los desechos en todo el Área Metropolitana de Buenos Aires, también influyeron en la motorización del circuito informal de recolección y recuperación, ya que al frenar seriamente la recuperación de desechos por la vía oficial, dejó el espacio para que dicha actividad fuera realizada por otros actores que estaban interesados en la compraventa de residuos, ya sea como estrategia de supervivencia o con objetivos comerciales. En este sentido, cabe decir que hasta el año 1997 la recolección de residuos de la ciudad de Buenos Aires estaba regulada por el Pliego 14/97 que disponía que sólo podía recuperarse hasta un $10 \%$ de los residuos recogidos y sólo durante la fase de recolección (Pliego 14/97), mientras que en el resto del Área Metropolitana el Decreto 9911/78 prohibía absolutamente la recuperación y/o reciclaje, y estipulaba que todos los desechos debían ser tratados por relleno sanitario. De este modo, y de acuerdo al artículo 11 del Decreto 9911/78 se prohibía la realización de cualquier tipo de tarea orientada a la recuperación de residuos, aún por quienes estuvieran a cargo de la recolección, es decir los municipios. De esta forma, y a diferencia de la ciudad de Buenos Aires, los municipios del Conurbano no tenían facultades para impulsar programas de recuperación, debido a que la ley los obligaba a trasladarlos a las plantas del CEAMSE (Coordinación Ecológica Área Metropolitana Sociedad del Estado), sin poder dar otro destino a sus desechos.

Unido a ello, el cambio en las pautas que presidían el escenario económico durante la década del '90 no hizo más que profundizar la situación, ya que al suprimirse la paridad cambiaria entre el peso argentino y el dólar estadounidense a principios del año 2002, se produjo una sustitución de insumos de fabricación que motorizó la cadena informal de recuperación. En forma paralela a esta sustitución de importaciones, se produjo un incremento de los precios de los materiales, que creció un 100\% entre el año 2001 y el 2002 (ESCLIAR et al., 2007).

En el contexto de esta conjugación de factores falta de empleo, desocupación creciente, disponibilidad de residuos en las calles de la ciudad y demanda de elementos reciclables por parte de las empresas la recolección informal de residuos se convirtió en una estrategia de supervivencia para muchas familias del Área Metropolitana de Buenos Aires.

En ese cuadro de situación, aumentó la cantidad de personas que ingresó al cirujeo y cambiaron las modalidades que exhibía tradicionalmente esta actividad.

\section{Nuevas modalidades de cirujeo en la ciudad de Buenos Aires a partir de mediados de la década de 1990}

Tal como expresé más arriba, si bien el cirujeo existió desde fines del siglo XIX, hacia mediados de la década del '90 se incrementó la cantidad de personas que ingresaron a la actividad y cambiaron los modos de realizar la tarea. ¿Quiénes son los "nuevos cirujas"?

Son habitantes del Área Metropolitana de Buenos Aires que ya eran pobres cuando empezó la crisis y se pauperizaron aún más cuando ésta eclosionó. Se trata de ex obreros de fábrica o de la construcción, repositores de supermercados, empleadas domésticas, zapateros o ex empleados de seguridad, que quedaron desocupados durante la década del '90 y que ante la falta de oportunidades de empleo en el mercado formal, encontraron en la recolección de residuos una estrategia de supervivencia para paliar la pobreza. Respecto de este tema, varios trabajos demuestran que la mayor parte de la población que "cirujeaba" en el año 2003 en la Ciudad de Buenos Aires lo hacía desde poco tiempo atrás (entre uno y dos años) y que previamente desarrollaban actividades no relacionadas con la recolección informal (PAIVA, 2008; PERELMAN, 2004; SUÁREZ, 2001). En este contexto aparece la categoría "cartonero" para expresar a este nuevo sujeto social, primero en los medios masivos de comunicación y luego generalizadamente en ámbitos estatales y académicos. Y en este escenario la ciudad de Buenos Aires, por ser gran generadora de desechos, se convierte en el centro de la actividad "cartonera".

En cuanto a los modos de acceso a la Ciudad si bien se registran formas muy diversas de transporte 
que incluyen el carro tirado a caballo, la bicicleta, o la camioneta, las modalidades paradigmáticas que se extendieron hacia fines de los '90 y en especial luego de la crisis 2001, fueron el tren y el camión.

En relación con el tren, en 1999 se inauguró la primera unidad específica para el traslado de cartoneros llamada "Tren Blanco" cuya particularidad era no poseer asientos para que pudieran viajar los cirujas con los carros. Dado el creciente número de personas que fueron ingresando a la actividad, desde fines de 2001 se formaron trenes cartoneros en todas las líneas de tren que acceden hasta la ciudad de Buenos Aires. Si bien sirvieron durante casi siete años, a principios del año 2008 se decretó el cierre de todos los trenes cartoneros, por iniciativa de las empresas privadas a cargo de estos transportes. Desde ese momento, muchas de las personas que se trasladaban en tren, lo hacen en camiones.

La segunda modalidad que se extendió durante el año 2001 fue el traslado en camión, esencialmente a través de tres tipologías: el "camión fletero", el "camión balanza” y el "camión empresa” (PARDO et al., 2006).

El "camión fletero" es el más extendido y transporta especialmente a los cartoneros que provienen de la zona sur del Conurbano Bonaerense. En estos casos, lo usual es que paguen al fletero un abono por el traslado en camión y que en muchas ocasiones el propio conductor se dedique también a la tarea de recolectar. Uno de los lugares de la ciudad de Buenos Aires en donde suelen concentrarse es el macrocentro, debido a que es un área de alta generación de desechos - en especial papel y cartón - por la gran cantidad de oficinas y locales de actividad comercial ubicados en la zona.

En cuanto al "camión balanza" su característica es que no traslada cartoneros, si no que sólo pesa y compra material, y en lo relativo al "camión empresa" se trata de un vehículo que transporta cartoneros que se dedican a recolectar a cambio de un jornal (PARDO et al., 2006).

En la urbe se recogen residuos revendibles como el cartón, el papel, los plásticos, los vidrios y metales, mientras que subsidiariamente se recogen los alimentos que entregan los comerciantes y la ropa que otorgan los vecinos.

Luego de la recolección, los cartoneros venden los residuos a intermediarios que actúan en el circuito informal de compraventa de desechos y que poseen diferente nivel de envergadura, en función del capital con que cuentan, el nivel de acopio que son capaces de alcanzar y las maquinarias que poseen. Según el único estudio realizado hasta el momento respecto de esta fase del circuito, para el año 2006 existían en la ciudad de Buenos Aires cerca de 35 galpones de distinto tamaño y capacidad de compra, muchos de los cuales se proveían directamente de los residuos que compran a los cartoneros y un sector reducido se abastecía directamente del rezago producido por los grandes generadores, tal como industrias gráficas y las fábricas que descartan grandes proporciones de papel o cartón.

El último eslabón de esta cadena de intermediación son las empresas que actúan en el mercado formal de la producción - papeleras, vidrierías, industrias plásticas o de envases - que se abastecen, en buena medida, de este circuito informal de recolección y recuperación. Como señalé al principio de este trabajo, en el año 2006, se tramitaba por este canal el 97\% de los desechos recuperados en la ciudad de Buenos Aires (PARDO et al., 2006).

No existen cifras exactas sobre la cantidad de personas que se encuentran "cartoneando" hacia el año 2012. La última estimación realizada por el Ministerio de Espacio Público del GCBA afirmaba que para el año 2008 existían aproximadamente unos 4900 recuperadores anotados en el registro oficial del Gobierno, mientras que para el año 2011 ese número había ascendido a 7600 personas (por supuesto, sólo las registradas) Es decir, que, en tres años, la cantidad de personas ascendió un 55\%. Dicho aumento se relaciona con un incremento de los precios de los materiales reciclables a nivel internacional y con el aumento de la cantidad de residuos diarios recogidos, debido a un incremento general del consumo de la población. De este modo, mientras que en el año 2008, por cada kilo de cartón vendido, un cartonero ganaba 0,20 centavos, en el año 2011 ganaba 0,60, además de subir de 110 a 200 kilos la cantidad que podía juntar diariamente (MORANDO, 2011).

Por las razones expuestas, desde la década de 1990 creció el cirujeo no organizado, pero además se conformaron varias cooperativas de recuperadores, muchas de las cuales desaparecieron y otras se encuentran actualmente integradas a la gestión oficial de los desechos.

En esta instancia corresponde recuperar la pregunta planteada inicialmente ¿Qué lectura ha realizado la teoría ambiental frente a un fenómeno como 
el descripto, que exhibe la paradoja de proteger el medio, al tiempo que descuida la calidad de vida de quienes lo llevan a cabo? Y en la misma línea, ¿qué posturas han tomado frente al tema los organismos encargados de planificar la política de gestión de los residuos en el Área Metropolitana de Buenos Aires?

\section{Las políticas públicas y la teoría ambiental frente al fenómeno de la recuperación informal de residuos}

Pese a la importancia del circuito informal en el proceso de recuperación de residuos, es posible afirmar que la teoría ambiental casi no se ha expedido sobre el fenómeno.

En lo concerniente a la teoría, se trata de un cuerpo de reciente formación nacido al calor de la noción de "ambiente humano" gestado en la Conferencia de Estocolmo sobre Medio Ambiente Humano de 1972 (NU, 1972) y reelaborado paulatinamente en función del concepto de Desarrollo Sustentable de los '90, que invoca la necesidad de sostener un desarrollo equilibrado entre el crecimiento económico, la sustentabilidad ecológica y el bienestar social (ALLEN, 1996) El principal objetivo de este cuerpo teórico es promover la intervención planificada sobre el ambiente, a través acciones encaminadas a conservar el equilibrio de la relación naturaleza-sociedad a través de un conjunto de herramientas pensadas "desde su origen" con un sentido ambiental (FERNÁNDEZ, 1996; GALLOPIN, 1986).

Dado que su meta es la planificación y la gestión sustentable, posee dificultades para analizar aquellas acciones que no nacieron con fines ambientalistas pero que impactan positivamente sobre el medio y menos aún aquellas que conjugan pobreza y ambiente justamente porque ponen en crisis uno de los aspectos centrales de esta posición que es el equilibro entre ambiente y sociedad y la preservación de la calidad de vida de los actores involucrados que es, además, un postulado fundamental del Desarrollo Sustentable.

En este contexto, sólo muy recientemente algunos autores latinoamericanos se han focalizado en la faceta ambiental de la actividad, ponderando el rol que cumplen los recolectores informales en la recuperación de desechos (DE LUCCA REIS COSTA, 2007; LOMBARDI, 2005; MEDINA, 1999; SCHAMBER, 2007; SUÁREZ, 2001). Se trata de autores de distintos países de Latinoamérica - México, Uruguay, Brasil, Argentina - formados en las ciencias sociales y en temas ambientales, que intentan aunar las realidades sociales de sus países con la cuestión ambiental y ponderar la acción de los recuperadores en la minimización de los residuos que llegan a disposición final. Sin embargo, por ahora se trata de estudios aislados que no han tomado posiciones relevantes dentro del cuerpo teórico existente sobre la gestión de los residuos sólidos urbanos.

En cuanto a los organismos encargados de planificar la política, desde el año 2002 hasta la actualidad se han tomado medidas que lentamente han ido incorporando a los cartoneros a la gestión oficial de los desechos, aunque con vaivenes.

Inicialmente fue el Gobierno de la ciudad de Buenos Aires quien tomó una posición activa frente al tema, sancionando en el año 2003 la Ley n. 992 que "incorpora a los recuperadores de residuos reciclables a la recolección diferenciada" (art. $2^{\circ}$ ) y crea el "Registro Único Permanente de Recuperadores de Materiales Reciclables" (art. 4º). (GCBA, 2003b). A partir de la sanción de esta Ley, se despenalizó la actividad en el ámbito de la ciudad de Buenos Aires, se formalizó la labor y se la integró como parte de la gestión oficial. Fundamentalmente, se intentó valorizar la tarea al resignificar la antigua figura del ciruja por la del "recuperador de residuos", brindándole, además, servicios médicos, elementos de higiene y seguridad (guantes) y credenciales identificadoras.

Cabe decir que el Gobierno de la Ciudad de Buenos Aires pudo llevar adelante esta política en el marco de la crisis 2001, es decir en un escenario muy favorable a la consagración de la problemática, ya que, en el contexto de aquella eclosión social y política, se dignificó la figura del cartonero asociándole valores positivos tales como la de "pobre pero digno" o "trabajador y honrado" (ADISSI, 2004, p. 5-8), lo que permitió instalar la temática en la escena pública y habilitó la sanción de leyes como la n. 992 que legalizó la actuación de los cartoneros y creó la figura del recuperador de residuos.

Desde aquella normativa que fue pionera en el tema, fueron sancionándose otras que siguieron el camino de la inclusión, aunque con idas y vueltas, y sin alcanzar a fijar una posición sostenida en torno del rol de los cartoneros en la gestión oficial.

En el año 2003, el Gobierno de la ciudad sancionó un nuevo pliego de recolección de residuos - 
el Pliego 6/03 - que puso en marcha la gestión integral de los residuos en la ciudad, habilitando la intervención de tres actores: las empresas privadas, los cartoneros y las cooperativas de recuperadores (GOBIERNO DE LA CIUDAD DE BUENOS AIRES - GCBA, 2003a). Dicho pliego establecía la creación de diversos centros verdes que serían administrados por cooperativas de recuperadores y que debían instalarse en cada una de las zonas de recolección de la ciudad. El rol de dichos centros sería funcionar como lugar de acopio, separación y clasificación de los residuos que llevaran allí los cartoneros que transitaban por la ciudad y realizar campañas de promoción ambiental entre los vecinos del barrio. Los centros verdes serían administrados por cooperativas de recuperadores que se sostendrían con lo recaudado de la venta de los residuos llevados a los centros.

Al calor de esta normativa se crearon diferentes centros verdes, como por ejemplo, la planta de reciclado de Janer y Varela gestionada por la Cooperativa Ecológica del Bajo Flores (CERBAF), el centro verde del barrio de Villa Soldati administrado por las cooperativas Reciclando Sueños y Del Oeste, el de la calle Varela al 2600 gestionado por la cooperativa "El Álamo", el ubicado en el barrio de Retiro administrado por "El Ceibo" y en el año 2009 otro en Barracas, cuya logística está a cargo de la cooperativa "Amanecer de los cartoneros" que pertenece al Movimiento de Trabajadores Excluidos (MTE). Paralelamente desde el año 2008 el Gobierno de la Ciudad de Buenos Aires (GCBA) realizó un convenio con los cartoneros agrupados en el Movimiento de Trabajadores Excluidos (MTE) por el cual dichos recolectores trabajan en diversas zonas de la ciudad de Buenos Aires con uniformes reglamentarios y percibiendo un ingreso que proviene de lo recolectado en la Ciudad (que venden en el sitio donde prefieran) y de un subsidio que les deposita el gobierno porteño mensualmente.

Desde la sanción de aquella normativa 992 que fue pionera en el tema de la inclusión de los cartoneros como recuperadores de residuos, el Gobierno de la Ciudad de Buenos Aires ha pasado por diferentes gestiones gubernamentales que si bien han sostenido la idea de incorporación de los cartoneros, han trabajado sobre criterios diferentes respecto del lugar que debe reservarse para ellos.
De este modo, mientras que la gestión de Aníbal Ibarra consagró la Ley n. 992 y sancionó el pliego de gestión de residuos n. 6/03 que hacia pié en tres actores: cartoneros no organizados, cooperativas y empresas privadas de recolección, la gestión iniciada por Mauricio Macri desde el año 2007 no es proclive a sostener el trabajo informal en las calles y persigue la idea de que todos los cartoneros se integren a cooperativas registradas por el GCBA. Desde esta postura realizó el convenio con el MTE para que los cartoneros trabajen con uniforme y con incentivos y favoreció la formación de nuevas cooperativas para que se hagan cargo de zonas de recolección.

En el año 2010 se llamo la licitación de un nuevo pliego de recolección en la ciudad de Buenos Aires dado que el 6/03 se encontraba vencido. Allí se estipuló la existencia de dos tipos de recolección de residuos: la de residuos húmedos a cargo de las empresas privadas y la de residuos secos reutilizables a cargo de las cooperativas que debían presentarse a licitación. Las cooperativas ganadoras alcanzan a trece más de las que ya existían y su rol será el de recolectar los residuos secos de los distintos barrios de la ciudad de Buenos Aires. Ello, por la vía del puerta a puerta o a través de los containers que ya funcionan en algunos barrios y que se pretenden instalar en toda la Ciudad de Buenos Aires.

En lo relativo al resto de los municipios del Área Metropolitana, en diciembre de 2006 se sancionó la Ley n. 13.596 de Gestión Integral de los Residuos Sólidos Urbanos (Ley Provincial 13.596, 2006) que rige para el resto de los municipios del Área Metropolitana de Buenos Aires, por la cual se procura la minimización, valorización, recuperación y/o reciclajes de los desechos y a partir de la cual - a diferencia de lo establecido por el Decreto 9911/78 los municipios tienen autonomía para decidir el destino final de sus desechos. De acuerdo a la nueva ley, cada municipio debió presentar un proyecto de gestión integral de los desechos, teniendo en cuenta la existencia de circuitos informales de recolección y recuperación con el fin de incorporarlos al sistema de gestión integral (art. 6을. Si bien la ley alerta a los municipios sobre la necesidad de tomar en cuenta la existencia de circuitos informales en sus planes de gestión de residuos sólidos, no los obliga a su inclusión, por lo cual es difícil establecer cuántos y cómo fueron integrados en los programas de recuperación y/o reciclado. 


\section{Comentarios finales}

Como se ha visto aquí, la ciudad de Buenos Aires sufre los problemas ambientales que viven las grandes ciudades del Primer Mundo, entre ellos, la excesiva generación de residuos sólidos urbanos (RSU). Pero tanto como sufre dichos problemas, también participa de las particularidades que exhiben las ciudades latinoamericanas en donde la ausencia de disposiciones públicas que regulen efectivamente el tratamiento diferenciado de los residuos, unido a la existencia de un alto índice de personas sin acceso al mercado formal de trabajo, genera que buena parte de la población se dedique a la recolección informal como estrategia de supervivencia.

En estas ciudades, lejos de las políticas puestas en marcha para los países desarrollados para minimizar y valorizar los residuos, son los pobres urbanos los que realizan, de hecho, las acciones tendientes a la recuperación de los RSU.

En Buenos Aires, este proceso es llevado adelante por un circuito informal de recolección, que si bien cuenta con una prolongada historia, adquiere una dimensión inusitada desde fines de los ‘90 y en especial luego de la crisis del 2001, ya que por esa vía se captan nada más y nada menos que el 97\% de los residuos sólidos urbanos recuperados en la ciudad de Buenos Aires.

A pesar de la potencia que posee este canal informal en la recolección y recuperación de residuos, hasta ahora la teoría ambiental ha prestado poca atención al tema. Como dije, se trata de un cuerpo teórico que nació para encaminar las acciones planificadas y racionales sobre el ambiente, y que, por esa razón, resulta escaso para dar cuenta de aquellas acciones que no nacieron con metas ambientalistas pero impactan positivamente sobre el medio. En ese marco sólo unos pocos autores latinoamericanos se han focalizado en la faceta ambiental de la actividad (BURIJSON; ROVERE; FOSSATI, 1998; DE LUCCA REIS COSTA, 2007; LOMBARDI, 2005; MEDINA, 1999; SCHAMBER, 2007; SUÁREZ, 2001) intentando incorporar la realidad social de sus ciudades a la gestión ambiental de los residuos.

En cuanto a las políticas tomadas por los organismos oficiales, es posible afirmar que luego de la crisis del año 2001 en que por primera vez el Gobierno de la Ciudad de Buenos Aires incluyó a los cartoneros dentro de la gestión oficial de los desechos a través de la
Ley n. 992 de recuperadores urbanos, los diferentes pliegos para la Ciudad, así como los distintos municipios provinciales han ido incorporando la problemática, aunque con posiciones oscilantes.

En lo relativo a la Ciudad de Buenos Aires en sus calles conviven cartoneros no organizados y otros semiformalizados a través de políticas que los incluyen dentro de la gestión, pero con mecanismos que no terminan de definir claramente cuál es el rol que se asigna a los cartoneros en la gestión de residuos, es decir, si se los tratará como pobres o trabajadores ambientales. Seguramente, esto se debe a que los cambios efectuados en las políticas públicas y la legislación se debieron más a la evidencia de la labor de los cartoneros en las calles y a una coyuntura de crisis social proclive a permitir su labor en la ciudad, que a una reflexión sistemática que pusiera en relación dicha práctica con la teoría ambiental y desde allí ordenara la gestión. De este modo, la única mención que aparece en las leyes y las políticas destinadas a organizar la tarea de los cartoneros es que los recuperadores son trabajadores ambientales, pero sin efectuar reflexiones más profundas en torno del significado y el alcance de la noción de "ambiente" y de las acciones destinadas a preservarlo.

Llegados a este punto vale reflexionar sobre algunas de las razones que han impedido que se tomen medidas definitivas sobre el rol de los cartoneros en la recuperación de residuos.

En estas breves conclusiones no pretendemos desplegar todas las aristas que rodean la cuestión, que son muchas y de diverso orden, pero sí, al menos, esbozar algunas líneas de análisis que permitan abrir la reflexión sobre el problema.

En principio, puede pensarse que la conflictiva relación "pobreza - ambiente" que acarrea la recolección informal de residuos y que entra en coalición con el propio concepto de ambiente - que desde los '70, resulta inseparable de la noción de calidad de vida - resulta una contradicción difícil de superar, que incide en la falta de acuerdo entre los decisores de políticas públicas.

En la misma medida, y no deslindado de lo anterior, si bien la crisis que estalló en el 2001 reconfiguró la imagen del cartonero en imaginario social y le asignó una serie de valores positivos tales como pobre pero digno, trabajador honrado o reciclador de residuos, lo cierto es que instaló la temática pero sin fijar un criterio definido en torno a su figura y 
al perfil de su labor, constituyendo un sujeto social que hasta hoy continúa con un estatus difuso en la estructura social. Concretamente, ¿qué es un cartonero en la mirada social? ¿Un pobre, un trabajador, un recuperador o un marginal?

En este sentido, tanto como en el imaginario popular, tampoco en el ámbito académico existe consenso para denotar el estatus social y laboral del cartonero. De este modo, mientras que en el 2002 el Instituto Nacional de Estadística y Censo (INDEC) los clasificó como "trabajadores informales", (INDEC, 2003) otros estudios analizan su labor como una "estrategia de supervivencia" (ROFMAN, 2004) y el sector ambientalista los denota como "recuperadores urbanos", dando muestra de la poca claridad que existe en torno al lugar social que ocupa el cartonero.

De acuerdo con ello, será difícil encontrar espacio definido para dichos actores en las políticas sobre gestión de residuos en tanto no quede claro en el imaginario social cuál es el valor social que asignamos a dicho sujeto y a la tarea que realiza.

Tal vez, una salida a esta situación sea renovar el debate que surgió al calor de la crisis 2001 y que luego se apagó. De avivarlo, sería deseable instalar el tema en el contexto más amplio de la discusión sobre el ambiente, su significado y las prácticas que lo preservan, de modo de que las políticas públicas surjan de debates amplios y no de la pura emergencia social, tal como fue el caso de las orientadas a la labor cartonera en el cuadro de la crisis del año 2001.

\section{Referencias}

ADISSI, G. El fenómeno cartonero en los medios gráficos porteños. La construcción de un nuevo sujeto objeto histórico. URBARED. 2004. Disponible en: <www.urbared. ungs.edu.ar>. Accedido el: 24 apr. 2005.

ALLEN, A. Introducción teórica al desarrollo urbano sustentable. Mar del Plata: Centro de Investigaciones Ambientales/UNMdP, 1996.

BECCARIA, L. Empleo e integración social. Buenos Aires: Fondo de Cultura Económica, 2001.

BURIJSON, V.; ROVERE, M.; FOSSATI, G. Reciclado de residuos sólidos urbanos en la ciudad de Buenos Aires. Buenos Aires: Gobierno de la ciudad de Buenos Aires, 1998.
DE LUCCA REIS COSTA, D. Márgenes en el centro. Calle, catación y basura en el centro de Sao Paulo. In: SUAREZ, F.; SCHAMBER, P. Recicloscopio: miradas sobre recuperadores urbanos de residuos en América Latina. Buenos Aires: Prometeo, Universidad de General Sarmiento, Universidad de Lanús, 2007. p. 47-61.

ESCLIAR, V. et al. Cartoneros ¿Una práctica individual o asociativa?: Ciudad de Buenos Aires, año 2004-2005. Buenos Aires: Centro Cultural de la Cooperación Floreal Gorini, 2007.

FERNÁNDEZ, R. Teoría de la Gestión Ambiental del Desarrollo Urbano. Mar del Plata: Centro de Investigaciones Ambientales, 1996.

GALLOPIN, G. Ecología y Ambiente. In: LEFF, E. (Comp.). Los problemas del conocimiento y la perspectiva ambiental del desarrollo. México: Siglo XXI, 1986. p.106-202.

GOBIERNO DE LA CIUDAD DE BUENOS AIRES - GCBA. Ordenanza n. 51453/97. Pliego de bases y condiciones por el cual se llama a licitación nacional e internacional para la recuperación y urbanización de la zona costera del Río de la Plata y sus modificaciones, circulares aclaratorias y contestación a las preguntas formuladas por los oferente. Boletín Oficial del Gobierno de la Ciudad - BOCBA, 1997.

GOBIERNO DE LA CIUDAD DE BUENOS AIRES - GCBA. Ley n. 992 de reglamentación del trabajo de los cartoneros, Reglamentada por Decreto n. 622 del Gobierno de la Ciudad. Buenos Aires. Boletín Oficial del Gobierno de la Ciudad - BOCBA, n. 1702, 2003a.

GOBIERNO DE LA CIUDAD DE BUENOS AIRES - GCBA. Decreto n. 1838. Pliego de Bases y condiciones generales y particulares para la licitación pública nacional e internacional para contratar la prestación del servicio público de higiene urbana para cinco zonas de la ciudad de Buenos Aires. Boletín Oficial del Gobierno de la Ciudad - BOCBA, n. 1795, 2003b.

GIDDENS, A. Action, structure, power. In: GIDDENS, A. Profiles and critiques in social theory. California: Press Berkeley and Los Angeles, 1982. p. 51-81.

HOSTIL, O. R. Content analysis for the social sciences and humanities. Massachusetts: Addison Wesley, 1969.

INSTITUTO NACIONAL DE ESTADÍSTICA Y CENSO INDEC. ¿Qué es el Gran Buenos Aires? 2003. Disponible en <http://www.indec.mecon.ar/nuevaweb/cuadros/1/ folleto\%20gba.pdf>. Accedido el: 7 agosto. 2012. 
INSTITUTO NACIONAL DE ESTADÍSTICA Y CENSO INDEC. Condiciones de vida - Línea de pobreza y canasta básica. 2007. Disponible en: <www. indec.mecon.ar/ principal.asp?id_tema=534>. Accedido el: 29 nov. 2008.

LOMBARDI, M. J. El reciclador marginado. Un análisis sobre la percepción de los residuos y los clasificadores informales. In: VI REUNIÓN DE ANTROPOLOGÍA DEL MERCOSUR, Montevideo, Uruguay, 2005. Actas... Montevideo: Facultad de Humanidades, Universidad de la República , 2005.

MEDINA, M. Reciclaje de residuos sólidos en América Latina. Frontera Norte, n. 21, 1999, p. 7-31.

MORANDO, L. Hay un 55\% más de cartoneros que en 2008 y recogen el doble de residuos. Diario Perfil, 12 jun. 2011. Disponible en: <http://www.perfil.com/ediciones/2011/6/edicion_580/contenidos/noticia_0057. html>. Accedido el: 2 agosto 2012.

NACIONES UNIDAS- NU. Conferencia de las Naciones Unidas sobre el Medio Ambiente y el Desarrollo. Río de Janeiro: Naciones Unidas. 1992. Disponible en: <www. un.org/esa/sustdev/documents/agenda21/spanish/ agenda21spchapter4.htm>. Accedido el: 29 enero 2008.

NACIONES UNIDAS - NU Conferencia de las Naciones Unidas sobre Medio Ambiente Humano. 1972. Disponible en: <http://www.prodiversitas.bioetica.org/ doc89.htm>. Accedido el: 6 agosto 2012.

PAIVA, V. El manejo formal e informal de los residuos sólidos urbanos de la ciudad de Buenos Aires entre los siglos XVI al XX. Área, Agenda de reflexión en Arquitectura, n. 14, 2008, p. 91-101.

PAIVA, V. Cartoneros y cooperativas de recuperadores: una mirada sobre la recolección informal de residuos. Área Metropolitana de Buenos Aires, 1999-2007. Buenos Aires: Prometeo, 2008.
PARDO, R. et al. Informe sobre el circuito del reciclado en la ciudad autónoma de Buenos Aires. Buenos Aires: Gobierno de la Ciudad de Buenos Aires, 2006.

PERELMAN, M. Algunas reflexiones en torno al caso del cirujeo en la ciudad de Buenos Aires. In: SEGUNDAS JORNADAS DE INVESTIGACIÓN EN ANTROPOLOGÍA SOCIAL, Buenos Aires, 2004. Actas... Buenos Aires: Facultad de Filosofía y Letras, Universidad de Buenos Aires, 2004.

RODRÍGUEZ VAL-LLOVERA, E. Gestión de residuos. Barcelona: Instituto de Estudios Ambientales para el Desarrollo Sostenible, 2003. p. 39-142.

ROFMAN, A. De piqueteros, cartoneros y trabajadores de empresas recuperadas: un modelo social emergencia en el espacio urbano argentino. Pobreza Urbana y Desarrollo, n. 24, v. 10, p. 25-50, 2004.

SCHAMBER, P. De los desechos a las mercancías. Antropología del reciclaje de residuos en el conurbano bonaerense. 2007. 283 f. Tesis Doctoral. Facultad de Filosofía y Letras, Universidad de Buenos Aires, Buenos Aires, 2007.

SUÁREZ, F. Actores Sociales de la Gestión de residuos sólidos en los partidos de Malvinas Argentinas y José C. Paz. 2001. 180 f. Tesis de maestría. Maestría en Políticas Ambientales y Territoriales - Facultad de Filosofía y Letras, Universidad de Buenos Aires, Buenos Aires, 2001.

VASILACHIS DE GIALDINO, I (Coord.). Estrategias de investigación cualitativa. Buenos Aires: Gedisa, 2007.

Recibido: 07/02/2013

Received: 02/07/2013

Aprobado: 14/04/2013

Approved: 04/14/2013 